\title{
Brachytelephalangic chondrodysplasia punctata
}

INSERM

\section{Source}

INSERM. (1999). Orphanet: an online rare disease and orphan drug data base.

Brachytelephalangic chondrodysplasia punctata. ORPHA:79345

Brachytelephalangic chondrodysplasia punctata (BCDP) is a form of non-rhizomelic chondrodysplasia punctata, a primary bone dysplasia, characterized by hypoplasia of the distal phalanges of the fingers, nasal hypoplasia, epiphyseal stippling appearing in the first year of life, as well as mild and non-rhizomelic shortness of the long bones. 